\title{
Multi-modal analysis on the intermittent contact dynamics of atomic force microscope
}

\author{
Y. Zhang ${ }^{\mathrm{a}, *}$, K.D. Murphy ${ }^{\mathrm{b}}$ \\ a State Key Laboratory of Nonlinear Mechanics (LNM), Institute of Mechanics, Chinese Academy of Sciences, Beijing 100190, China \\ ${ }^{\mathrm{b}}$ Department of Mechanical Engineering, University of Connecticut, Storrs, CT 06268-3139, USA
}

\section{A R T I C L E I N F O}

\section{Article history:}

Received 14 April 2011

Received in revised form

28 June 2011

Accepted 17 July 2011

Handling Editor: L.G. Tham

Available online 3 August 2011

\begin{abstract}
A B S T R A C T
A multi-modal analysis on the intermittent contact between an atomic force microscope (AFM) with a soft sample is presented. The intermittent contact induces the participation of the higher modes into the motion and various subharmonic motions are shown. The AFM tip mass enhances the coupling of different modes. The AFM tip mass is modeled by the Dirac delta function and the coupling effects are analyzed via the Galerkin method. The necessity of applying multi-modal analysis to the intermittent contact problem is demonstrated. Unlike the impact oscillator model which assumes the impact/contact time is infinitesimal, the contact time can be a significant fractional portion in each cycle, especially for the soft sample case and thus results in different dynamic behavior from that of an impact oscillator.
\end{abstract}

(c) 2011 Elsevier Ltd. All rights reserved.

\section{Introduction}

Atomic force microscope (AFM) has been a very useful tool in many fields since its inception in 1986 [1]. One of the AFM operatingmodes is called vibrating or tapping mode [2,3], which can reduce shear [4] and it can thus be used for soft materials to prevent or reduce the damage. For example, AFM is used to measure the cell wall nanomechanical motion of the living Saccharomyces cerevisiae (baker's yeast) [5]. The intermittent contact between the AFM tip and sample often occurs in the tapping mode. Since Burham et al. [6] first linked the AFM intermittent contact dynamics to an impact oscillator model developed by Pippard for a pin hitting a loudspeaker [7], many theories/models have been developed [3,4,8-13]. In those studies [3,4,8-13], either one degree-of-freedom (DOF) model of a spring-mass system as shown in Fig. 1 or the single mode analysis is applied to the AFM cantilever of a continuous system. One DOF model or single mode analysis can be accurate in some special cases. For example, an AFM vibrates with a driving frequency lower than or around the first natural frequency of the AFM cantilever and has no contact with the sample [14]. However, the driving frequency can be much higher than the first natural frequency of AFM cantilever [6,15] and the higher modes can thus participate in the motion. In the AFM imaging application, the phase shift of higher mode has been used to characterize the mechanical, electrical and magnetic interactions [15]. We demonstrate that the results computed by the single mode analysis can be very different from those computed by multimodes when the driving frequency is higher than the AFM cantilever first natural frequency. Even when the driving frequency is less than the AFM first natural frequency, the higher modes can still participate in the motion due to the repulsive tip-sample interaction of impact [16]. The repulsive tipsample interaction has much shorter acting range and time than those of the attractive ones such as van der Waals (vdW)

\footnotetext{
* Corresponding author. Tel.: +8610825 43970.

E-mail address: zhangyin@lnm.imech.ac.cn (Y. Zhang).
} 
(a)

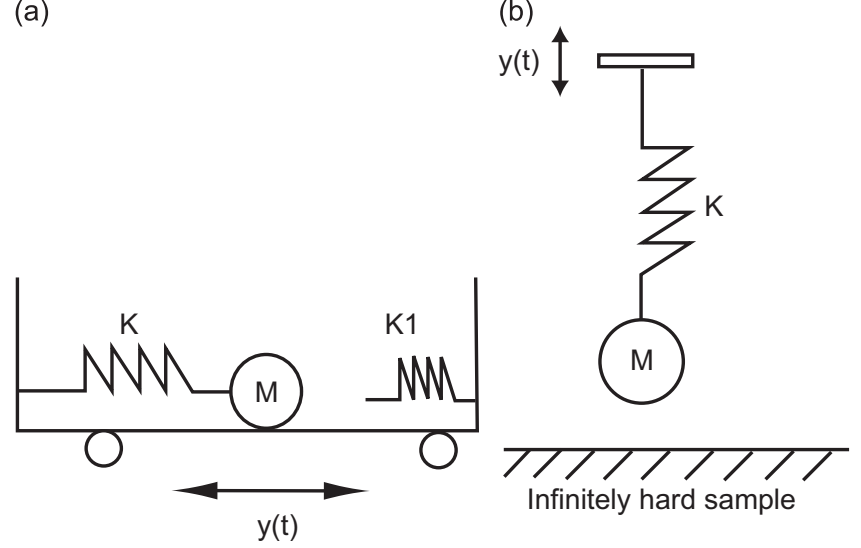

Fig. 1. Schematic diagrams of one DOF modeling. (a) The vibro-impact oscillator model; (b) impact oscillator.

and Casimir forces. The repulsive tip-sample interaction acts as an impulse to excite the higher modes[16]. The total harmonic distortion (THD) is used to measure the fraction of power transferred from the fundamental into the higher harmonics and THD ranges from 0 to 100 percent. THD with the maximum value of 15 percent is observed when an AFM is driven at the first natural frequency [16]. The higher modes participation in the motion has been fully recognized in the vibration of scanning acoustic microscope, which is also a cantilever structure and has stronger tip-sample interaction [17]. Attard et al. [13] noticed that the tip mass can significantly contribute to the whole system inertia. The concentrated mass not only affects the system natural frequencies but also couples the (orthogonal) modes as reflected by the Dirac delta function used to model the concentrated mass even for the linear small vibration case [18,19]. Furthermore, the geometrical nonlinearity of large vibration amplitude can also couple the modes [20]. The multi-modal analysis rather than one DOF model or single mode analysis should be the general approach for the study of the AFM intermittent contact dynamics. The reasons for this can be summarized as the following three: (1) that the AFM driving frequency can be higher than the first natural frequency; (2) that the repulsive tip-sample interaction can induce the motion of higher modes; (3) mode coupling due to the concentrated tip mass or the geometrical nonlinearity of large motion.

When the stiffness $K_{1}$ of the vibro-impact system as shown in Fig. 1a approaches infinity or a mass-spring system hits an infinitely hard substrate as shown in Fig. 1b, the system becomes an impact oscillator [21]. The impact of an impact oscillator is instantaneous and the instantaneous reflection velocity is only related to the instantaneous velocity just before the impact via the coefficient of restitution [3,21]. The coefficient of restitution is also responsible for the major energy loss in an impact oscillator system. The challenges of applying the impact oscillator model to the AFM intermittent contact dynamics are recognized and summarized as the following two questions by van de Water and Molenaar [3]: Is the energy loss concentrated at the impact instance and is the impact instantaneous? The contact time is dependent on the driving frequency/amplitude, the bending and contact stiffnesses of AFM and surface, etc. [22]. The interaction/contact time of the tapping-mode AFM and sample surface in general is a considerable fraction of the cycle time [16]. Besides the coefficient of restitution, the hysteresis due to the adhesive forces can also cause the energy dissipation [9]. van de Water and Molenaar [3] demonstrated that for the grazing impact, the tapping-mode AFM displays the characteristic features of an impact oscillator. However, the grazing impact is a particular impact case with zero impact velocity [22,23]. The AFM studied by van de Water and Molenaar interacts with the rigid surface through a liquid bridge, which offers a mechanism to realize the grazing impact [3]. However, the zero velocity impact is not general in the AFM intermittent contact dynamics. In this study the contact between an AFM tip and a surface is modeled as the AFM hitting a linear spring and a damper. The relatively small spring stiffness is chosen to model the soft sample and the contact time can thus be a significant fraction of the period. We demonstrate that the bifurcation diagram and phase portraits of the system are very different from those of an impact oscillator. The more general criterion of using the AFM tip displacement $[8,12]$ to tell whether the contact occurs is also adopted in this study.

The subharmonics and chaos are the two characteristic features in the intermittent contact dynamics of both the macroscopic structure [7,21,24-29] and the microstructure (of AFM) [3,6,9]. Measuring the AFM frequency shift has been demonstrated as an effective method to determine the tip-sample potential [30,31]. However, in those frequency shift theories/models there is an implicit assumption that the AFM subharmonic motion does not occur. When the AFM tipsample distance is in the order of interatomic spacing [31], the very strong tip-sample interaction due to Lennard-Jones potential can induce not only the aforementioned higher modes participation in motion but also the subharmonic motion, which can cause great trouble in the frequency shift theories/models. For a microstructure, the contribution of the adhesion, vdW and Casimir forces [8,14,32] can be significant to the system dynamics and stability. For an AFM in the noncontact tapping mode, the vdW force is responsible for the system frequency shift and softening effect [14]. However, for the AFM intermittent contact in tapping mode, the impact/contact itself is of the primary importance to the AFM dynamic behavior $[3,9]$. The nonlinearity of impact/contact forces is of the secondary importance. Although adhesion 
induces nonlinear contact behavior, the adhesion influence is rather weak as compared with an elastic one [33]. The adhesion influence only stands out when the external mechanical load is very small [33] and the elastic force due to impact is dominant in the AFM intermittent dynamics. Because the long-range attractive vdW force does not contribute to the energy loss of the tapping-mode AFM, its influence on the AFM dynamics is ignored [3,9]. This study is to investigate the AFM intermittent dynamics with more than one mode participation and the interaction with a relatively soft sample. The secondary influences of adhesion, vdW and Casimir forces are thus not included.

\section{Model development}

In Fig. 2a, the coordinate system and AFM cantilever dimensions are shown. The AFM cantilever and its tip are separated from the sample substrate with the distances of $g_{0}$ and $g_{1}$, respectively. AFM is driven with a forced motion $y(t)(t$ is time) at its fixed end. $w(x, t)$ in Fig. $2 \mathrm{~b}$ is the beam deflection measured from the fixed end. When the AFM tip hits the sample (Fig. 2c), the AFM tip is in contact with a spring with stiffness $K_{1}$ and a damper with the viscous damping of $C_{1}$ as seen in Fig. $2 \mathrm{~d}$. The spring stiffness $K_{1}$ physically corresponds to the contact stiffness between the AFM tip and substrate and its value depends on the tip geometry, Young's moduli and Poisson's ratios of the tip and substrate [33]. $C_{1}$ can be obtained by measuring the energy dissipated into the sample per cycle [34]. Fig. 2e shows the first three modes of cantilever beam. Because of the participation of higher modes into the motion, it is well possible that the other part of AFM rather than the tip is in contact with the sample as shown in Fig. 2f. If such scenario occurs, it is a rather difficult task to compute the contact area and equilibrium [35]. We assume that such contact scenario does not happen. During the computation, the driving frequency/ amplitude and other related parameters are carefully chosen and examined to avoid such contact scenario.

The cantilever beam displacement $v(x, t)$ in the coordinate system shown in Fig. 2a is

$$
v(x, t)=w(x, t)+y(t)+g_{0},
$$

the beam tip displacement $v_{T}(x, t)$ is

$$
v_{T}(x, t)=v_{T}(t)=v(L, t)-\left(g_{0}-g_{1}\right)=w(L, t)+y(t)+g_{1} .
$$

For brevity, the following equation of motion is given, which is derived by applying the Hamilton principle [19]

$$
\begin{cases}{[M \delta(x-L)+m] \frac{\partial^{2} v}{\partial t^{2}}+C \frac{\partial v}{\partial t}+E I \frac{\partial^{4} v}{\partial x^{4}}=0,} & v_{T}>0 \\ {[M \delta(x-L)+m] \frac{\partial^{2} v}{\partial t^{2}}+C \frac{\partial v}{\partial t}+E I \frac{\partial^{4} v}{\partial x^{4}}+K_{1} \delta(x-L)\left[w(x, t)+y(t)+g_{1}\right]+C_{1} \delta(x-L) \frac{\partial v}{\partial t}=0,} & v_{T} \leq 0 .\end{cases}
$$

(a)

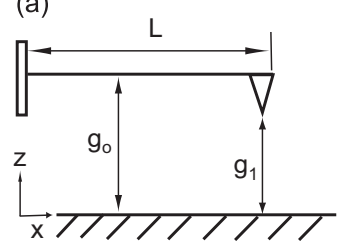

(c)

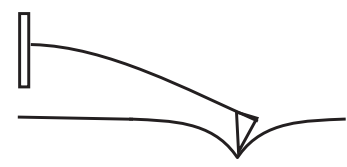

(e)

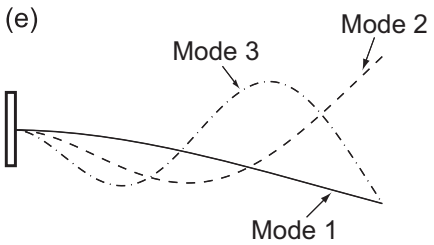

(b)

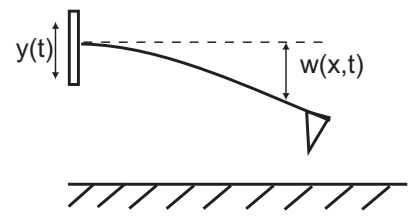

(d)

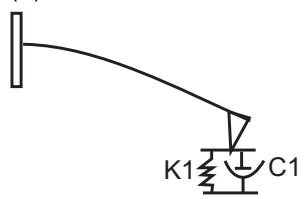

Fig. 2. Schematic diagrams of the cantilever beam with a concentrated mass at the free end. (a) The beam (neutral axis) and the tip are initially separated from the substrate with the gap distances of $g_{0}$ and $g_{1}$, respectively. The coordinate system is also shown. (b) $y(t)$ is the oscillation of the beam fixed end and $w(x, t)$ is the beam deflection from the fixed end. (c) The beam tip hits the substrate when the tip displacement reaches a certain value. (d) When the tip is in contact with the substrate, we model the beam tip hits a spring with the stiffness of $K_{1}$ and a damper with the viscous damping of $C_{1}$. (e) The lowest three mode shapes are shown. (f) With the participation of higher mode shapes participate, the beam part other than the AFM tip is in contact with sample, which is the contact with a finite length rather than a point contact. 
$M$ is the tip mass and $m$ is the mass per unit length of the beam. $C$ is the viscous damping of the beam. $E$ is the beam Young's modulus and $I=b h^{3} / 12$ ( $b$ : beam width, $h$ : beam height) for a rectangular beam. Here $\delta$ is the Dirac delta function. $K_{1}$ is the spring stiffness and $C_{1}$ is the viscous damping due to the tip-sample contact. $v_{T}$ defines the switching condition $[8,21,26,27] . v_{T}$ is a function of time $t, v_{T}=0$ indicates that the AFM tip starts the contact with the sample, or say, impacts the sample. However, keep in mind that the contact/impact time is unknown. Although the analytical solutions before and after impact are available to the piecewise linear impact oscillator, the unknown property of impact time makes the system nonlinear [26,22]. $y(t)$ is a sinusoidal driving motion given as follows:

$$
y(t)=f \sin (\omega t) .
$$

$f$ and $\omega$ are the driving amplitude and frequency, respectively. The following dimensionless quantities are introduced to nondimensionalize equation (3)

$$
\xi=\frac{x}{L}, \quad \tau=\sqrt{\frac{E I}{m L^{4}}} t, \quad W=\frac{w}{g_{0}}, \quad V=\frac{v}{g_{0}}, \quad V_{T}=\frac{v_{T}}{g_{0}}, \quad \Omega=\sqrt{\frac{m L^{4}}{E I}} \omega .
$$

$\sqrt{E I / m L^{4}}$ is with the unit of Hertz and it is the same order of the first natural frequency of uniform and undamped cantilever beam [36]. The governing equations of Eq. (3) now become as follows:

$$
\left\{\begin{array}{cr}
{\left[\alpha_{1} \delta(\xi-1)+1\right] \frac{\partial^{2} W}{\partial \tau^{2}}+\alpha_{3} \frac{\partial W}{\partial \tau}+\frac{\partial^{4} W}{\partial \xi^{4}}=\left[\alpha_{1} \delta(\xi-1)+1\right] \alpha_{2} \Omega^{2} \sin (\Omega \tau)-\alpha_{2} \alpha_{3} \Omega \cos (\Omega \tau),} & V_{T}>0 \\
{\left[\alpha_{1} \delta(\xi-1)+1\right] \frac{\partial^{2} W}{\partial \tau^{2}}+\alpha_{3} \frac{\partial W}{\partial \tau}+\frac{\partial^{4} W}{\partial \xi^{4}}+\alpha_{4} \delta(\xi-1) W+\alpha_{6} \delta(\xi-1) \frac{\partial W}{\partial \tau}=\left[\alpha_{1} \delta(\xi-1)+1\right] \alpha_{2} \Omega^{2} \sin (\Omega \tau)} & V_{T} \leq 0 \\
-\alpha_{2} \alpha_{3} \Omega \cos (\Omega \tau)-\alpha_{2} \alpha_{4} \delta(\xi-1) \sin (\Omega \tau)-\alpha_{4} \alpha_{5} \delta(\xi-1)-\alpha_{2} \alpha_{6} \delta(\xi-1) \Omega \cos (\Omega \tau), &
\end{array}\right.
$$

Here $\alpha_{i}$ 's $(i=1-6)$ are defined as

$$
\alpha_{1}=\frac{M}{m L}, \quad \alpha_{2}=\frac{f}{g_{0}}, \quad \alpha_{3}=C \sqrt{\frac{L^{4}}{E I m}}, \quad \alpha_{4}=\frac{K_{1} L^{3}}{E I}, \quad \alpha_{5}=\frac{g_{1}}{g_{0}}, \quad \alpha_{6}=\frac{C_{1}}{L} \sqrt{\frac{L^{4}}{E I m}} .
$$

Physically $\alpha_{1}$ indicates the ratio of the tip mass to the whole beam mass and $\alpha_{4}$ indicates the ratio of the spring stiffness to the beam bending stiffness. $\alpha_{2}$ and $\alpha_{5}$ indicate the relationship between driving amplitude and tip separation distance with the beam separation distance, respectively. $\alpha_{3}$ and $\alpha_{6}$ indicate the damping influence.

The switching condition of Eq. (2) is now nondimensionalized as follows:

$$
V_{T}=W(1, \tau)+\frac{f}{g_{0}} \sin (\Omega \tau)+\frac{g_{1}}{g_{0}}=W(1, \tau)+\alpha_{2} \sin (\Omega \tau)+\alpha_{5} .
$$

Here the Galerkin method is used for the computation of Eq. (6) and $W(\xi, \tau)$ is discretized as

$$
W(\xi, \tau)=\sum_{j=1}^{N} a_{j}(\tau) \phi_{i}(\xi)
$$

$a_{j}(\tau)$ is the unknown modal amplitude to be determined and $N$ is the mode number. $\phi_{j}(\xi)$ is the mode shape of cantilever beam given by Chang and Craig [36]. Substitute Eq. (9) into Eq. (6), time $\phi_{i}(\xi)$ and integrate from 0 to 1 , the following governing equations are derived:

$$
\begin{cases}{\left[\mathbf{M}^{\mathbf{I}}\right] \ddot{\mathbf{X}}+\left[\mathbf{C}^{\mathbf{I}}\right] \dot{\mathbf{X}}+\left[\mathbf{K}^{\mathbf{I}}\right] \mathbf{X}=\mathbf{F}^{\mathbf{I}},} & V_{T}>0 \\ {\left[\mathbf{M}^{\mathbf{I I}}\right] \ddot{\mathbf{X}}+\left[\mathbf{C}^{\mathbf{I I}}\right] \dot{\mathbf{X}}+\left[\mathbf{K}^{\mathbf{I I}}\right] \mathbf{X}=\mathbf{F}^{\mathbf{I I}},} & V_{T} \leq 0 .\end{cases}
$$

Here $\dot{O}=\partial / \partial \tau, \mathbf{X}=\left(a_{1}, a_{2}, \ldots, a_{N}\right)^{\mathrm{T}}, \mathbf{F}^{I}=\left(F_{1}^{I}, F_{2}^{I}, \ldots, F_{N}^{I}\right)^{\mathrm{T}}$ and $\mathbf{F}^{\mathrm{II}}=\left(F_{1}^{I I}, F_{2}^{I I}, \ldots, F_{N}^{I I}\right)^{\mathrm{T}}$. During the integration, the orthogonality

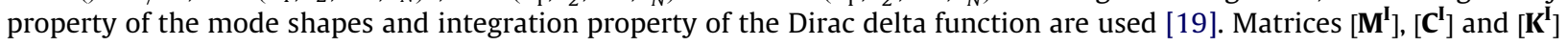
are derived as follows:

$$
\begin{gathered}
{\left[\mathbf{M}^{\mathbf{I}}\right]_{i j}= \begin{cases}\alpha_{1} \phi_{i}(1) \phi_{i}(1)+\int_{0}^{1} \phi_{i}(\xi) \phi_{i}(\xi) \mathrm{d} \xi, & i=j, \\
\alpha_{1} \phi_{i}(1) \phi_{j}(1), & i \neq j,\end{cases} } \\
{\left[\mathbf{C}^{\mathbf{I}}\right]_{i j}= \begin{cases}\alpha_{3} \int_{0}^{1} \phi_{i}(\xi) \phi_{i}(\xi) \mathrm{d} \xi, & i=j, \\
0, & i \neq j,\end{cases} }
\end{gathered}
$$

and

$$
\left[\mathbf{K}^{\mathbf{I}}\right]_{i j}= \begin{cases}\int_{0}^{1} \phi_{i}(\xi) \frac{\partial^{4} \phi_{i}(\xi)}{\partial \xi^{4}} \mathrm{~d} \xi, & i=j \\ 0, & i \neq j\end{cases}
$$


Clearly due to the integration property of the Dirac delta function, $\left[\mathbf{M}^{\mathbf{l}}\right]$ is not diagonal, which means the coupling of modes even for small linear vibration. Vector $\mathbf{F}^{\mathbf{I}}$ is defined as

$$
\mathbf{F}_{i}^{\mathbf{I}}=\alpha_{2} \Omega^{2}\left[\alpha_{1} \phi_{i}(1)+\int_{0}^{1} \phi_{i}(\xi) \mathrm{d} \xi\right] \sin (\Omega \tau)-\alpha_{2} \alpha_{3} \int_{0}^{1} \phi_{i}(\xi) \mathrm{d} \xi \cos (\Omega \tau) .
$$

Matrices $\left[\mathbf{M}^{\mathbf{I I}}\right],\left[\mathbf{C}^{\mathbf{I I}}\right]$ and $\left[\mathbf{K}^{\mathbf{I I}}\right]$ are given as follows:

$$
\begin{gathered}
{\left[\mathbf{M}^{\mathbf{I I}}\right]=\left[\mathbf{M}^{\mathbf{I}}\right],} \\
{\left[\mathbf{C}^{\mathbf{I I}}\right]_{i j}= \begin{cases}\alpha_{3} \int_{0}^{1} \phi_{i}(\xi) \phi_{i}(\xi) \mathrm{d} \xi+\alpha_{6} \phi_{i}(1) \phi_{i}(1), & i=j, \\
\alpha_{6} \phi_{i}(1) \phi_{j}(1), & i \neq j,\end{cases} }
\end{gathered}
$$

and

$$
\left[\mathbf{K}^{\mathbf{I I}}\right]_{i j}= \begin{cases}\int_{0}^{1} \phi_{i}(\xi) \frac{\partial^{4} \phi_{i}(\xi)}{\partial \xi^{4}} \mathrm{~d} \xi+\alpha_{4} \phi_{i}(1) \phi_{i}(1), & i=j, \\ \alpha_{4} \phi_{i}(1) \phi_{j}(1), & i \neq j,\end{cases}
$$

Clearly the spring/damper also couples the mode shapes due to the Dirac delta function. $\mathbf{F}^{\text {II }}$ is given as follows:

$$
\mathbf{F}_{i}^{\mathbf{I I}}=\mathbf{F}_{i}^{\mathbf{I}}-\alpha_{2} \alpha_{4} \phi_{i}(1) \sin (\Omega \tau)-\alpha_{4} \alpha_{5} \phi_{i}(1)-\alpha_{2} \alpha_{6} \phi_{i}(1) \Omega \cos (\Omega \tau) .
$$

The fourth-order Runge-Kutta integration is used for the time integration of Eq. (10) [37]. As indicated in Eq. (10), the natural frequencies of the systems before and after contact can be very different depending on $\alpha_{4}$. To achieve the same accuracy for the system before and after contact, the time step is different. It is well possible that the integration routine with large time step can overshoot the discontinuity to cause the computational inaccuracy [38]. To avoid such scenario is either to construct the time grid which reduces dramatically as an AFM/oscillator approaches the spring and keeps small during contact [38] or just to take very small time step for both contact and noncontact regions.

\section{Eigenfrequencies and mode coupling}

The homogeneous parts of Eq. (10) are

$$
\begin{cases}{\left[\mathbf{M}^{\mathbf{I}}\right] \ddot{\mathbf{X}}+\left[\mathbf{C}^{\mathbf{I}}\right] \dot{\mathbf{X}}+\left[\mathbf{K}^{\mathbf{I}}\right] \mathbf{X}=0,} & V_{T}>0 \\ {\left[\mathbf{M}^{\mathbf{I}}\right] \ddot{\mathbf{X}}+\left[\mathbf{C}^{\mathbf{I}}\right] \dot{\mathbf{X}}+\left[\mathbf{K}^{\mathbf{I}}\right] \mathbf{X}=0,} & V_{T} \leq 0\end{cases}
$$

For the natural frequency of the system, we only need to study the contact part because the noncontact part is the particular case of $\alpha_{4}=\alpha_{6}=0$. The eigenvalue problem can be formed as the following form [39]:

$$
\left[\mathbf{M}^{*}\right] \dot{\mathbf{Q}}(\tau)+\left[\mathbf{K}^{*}\right] \mathbf{Q}(\tau)=0
$$

$\left[\mathbf{M}^{*}\right],\left[\mathbf{K}^{*}\right]$ and $\mathbf{Q}(\tau)$ are defined as

$$
\left[\mathbf{M}^{*}\right]=\left(\begin{array}{cc}
{\left[\mathbf{M}^{\mathbf{I I}}\right]} & \mathbf{0} \\
\mathbf{0} & {\left[-\mathbf{K}^{\mathbf{I I}}\right]}
\end{array}\right), \quad\left[\mathbf{K}^{*}\right]=\left(\begin{array}{cc}
{\left[\mathbf{C}^{\mathbf{I I}}\right]} & {\left[\mathbf{K}^{\mathbf{I I}}\right]} \\
{\left[\mathbf{K}^{\mathbf{I I}}\right]} & \mathbf{0}
\end{array}\right), \quad \mathbf{Q}=\left(\dot{\mathbf{X}}(\tau)^{\mathrm{T}} \mathbf{X}(\tau)^{\mathrm{T}}\right)
$$

The eigenfrequencies of the beam in contact can be computed from Eq. (20). In order to compare the results with the eigenfrequencies of a uniform and undamped cantilever beam obtained by Chang and Craig [36], $\alpha_{3}$ and $\alpha_{6}$ are set zero, and the dimensionless frequency $\omega_{0 i}$ is defined as

$$
\omega_{0 i}=\sqrt{\frac{m L^{4}}{E I}} f_{i}
$$

$f_{i}$ is the $i$ th dimensional eigenfrequency of a uniform and undamped cantilever beam, which is the case of $\alpha_{1}=\alpha_{3}=\alpha_{4}=\alpha_{6}=0 . \omega_{0 i}$ is given by Chang and Craig [36]. Here we give the first three of them

$$
\omega_{01}=1.875^{2}=3.52, \quad \omega_{02}=4.694^{2}=22.03, \quad \omega_{03}=7.855^{2}=61.7 .
$$

The first two natural frequencies of Eq. (20) as functions of $\alpha_{1}$ and $\alpha_{4}$ are presented in Fig. 3. They are divided by $\omega_{01}$ and $\omega_{02}$ to show the relative change. Clearly $\alpha_{1}$ and $\alpha_{4}$ have larger influences on the system first natural frequency $\omega_{1}$. It is also worth mentioning that the eigenfrequency for this intermittent contact system is amplitude-independent, which is not usual in a nonlinear system [27]. The discontinuity of eigenfrequencies, or say, the discontinuity of stiffness, which is embodied in switching condition, is responsible for the nonlinearity of the intermittent contact system with small vibration amplitude here. For one DOF system, the natural frequency discontinuity before and after contact can be directly incorporated in the equation of motion [26]. However, Fig. 3 does not tell any information on the mode coupling. To show 

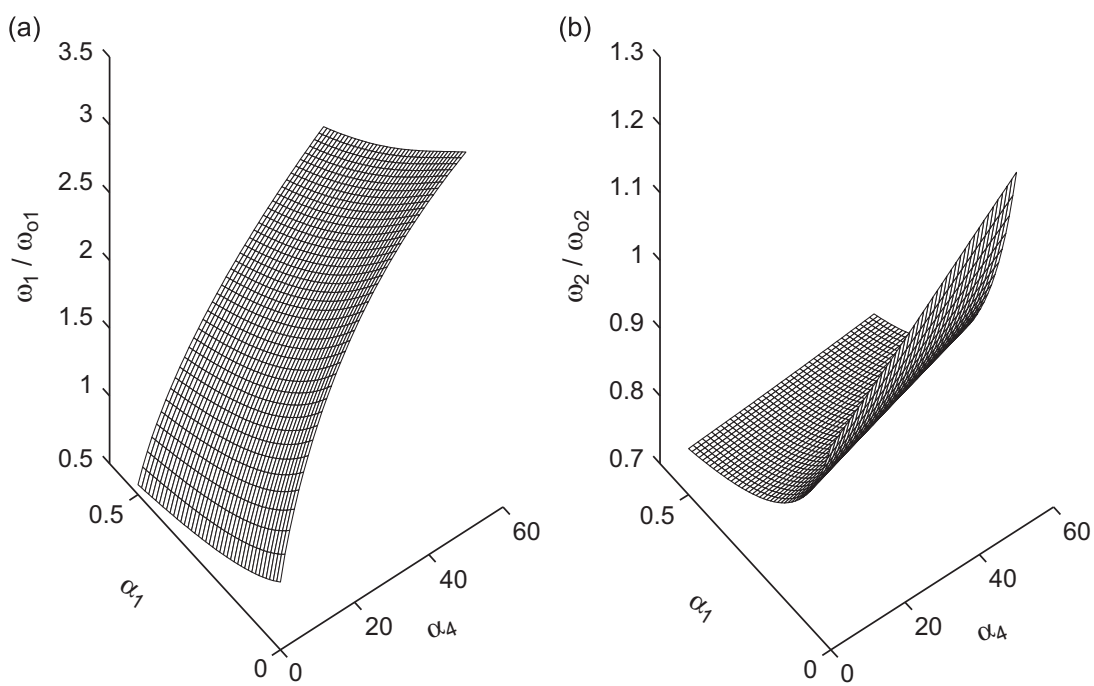

Fig. 3. The changes of the natural frequencies as the functions of $\alpha_{1}$ and $\alpha_{4}$. (a) The first natural frequency change; (b) the second natural frequency change.

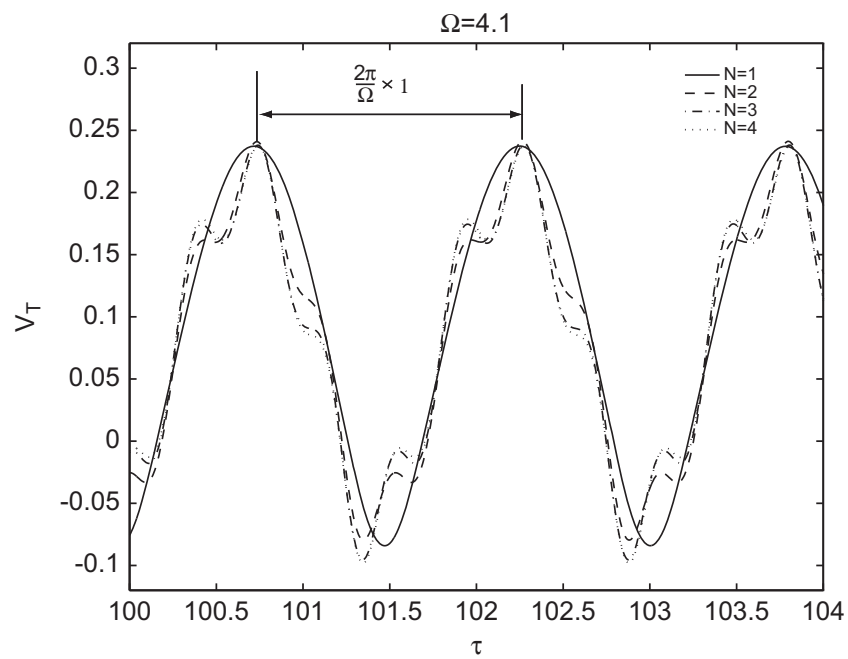

Fig. 4. The convergence study on the mode number when $\Omega=4.1$ and $\alpha_{1}=0.1$. The mode number is taken as $1,2,3$ and 4 , respectively.

the mode coupling effect, we present an example here. The parameters are given as follows:

$$
\alpha_{1}=\alpha_{2}=0.1, \quad \alpha_{3}=\alpha_{5}=\alpha_{6}=0, \quad \alpha_{4}=20 .
$$

$\alpha_{5}$ here is set to be zero to make sure that the system can always have the intermittent contact because it is well possible that the system does not have any contact under some driving frequency and amplitude for nonzero $\alpha_{5}$ [28]. With the parameters in Eq. (24), the three eigenfrequencies of the system in contact are

$$
\omega_{1}=7.79, \quad \omega_{2}=20.33, \quad \omega_{3}=55.81 .
$$

It is interesting to notice that compared with the eigenfrequencies without contact in Eq. (23), $\omega_{1}$ increases dramatically, while both $\omega_{2}$ and $\omega_{3}$ reduce. The driving frequency $\Omega$ is taken as 4.1, which is between $\omega_{01}$ and $\omega_{1}$, but far from the second and third eigenfrequencies of the system with or without contact. At a first look, the first mode should be dominant in the motion at $\Omega=4.1$ for both free-play and contacting vibrations. If it is true, single mode analysis should be accurate enough to describe the motion. While, the convergence study as shown in Fig. 4 disagrees with this conclusion. The tip motion in Fig. 4 is the steady-state motion. Clearly the motion computed by the single mode $(N=1)$ has a significant difference with those of $N=2,3,4$. For $N=3,4$, there is very little difference in the motion. The second and third modes participation in the motion induced by the intermittent contact are responsible for the difference. Fig. 4 demonstrates that even for the system under the low driving frequency which is far from the second and third eigenfrequencies, the higher 
modes $(N=2,3)$ can still be excited by the intermittent contact. As $V_{T} \leq 0$ indicates that the tip is in contact with sample, it can be seen from Fig. 4 that the contact time is a significant portion of the cycle. This can make the dynamic behavior of the system quite different from that of an impact oscillator.

\section{Results and discussions}

Subharmonic is the period- $n$ oscillation that takes $n$ forcing periods to complete a full cycle [40,41]. Subharmonic is the structurally stable type of bifurcation [42], which physically means that when the perturbation is qualitatively similar, the system will have similar dynamic behavior (phase portrait).

In Figs. 5-17, the following parameters are fixed:

$$
\alpha_{2}=0.1, \quad \alpha_{3}=0.1, \quad \alpha_{4}=20, \quad \alpha_{5}=0, \quad \alpha_{6}=0.2
$$

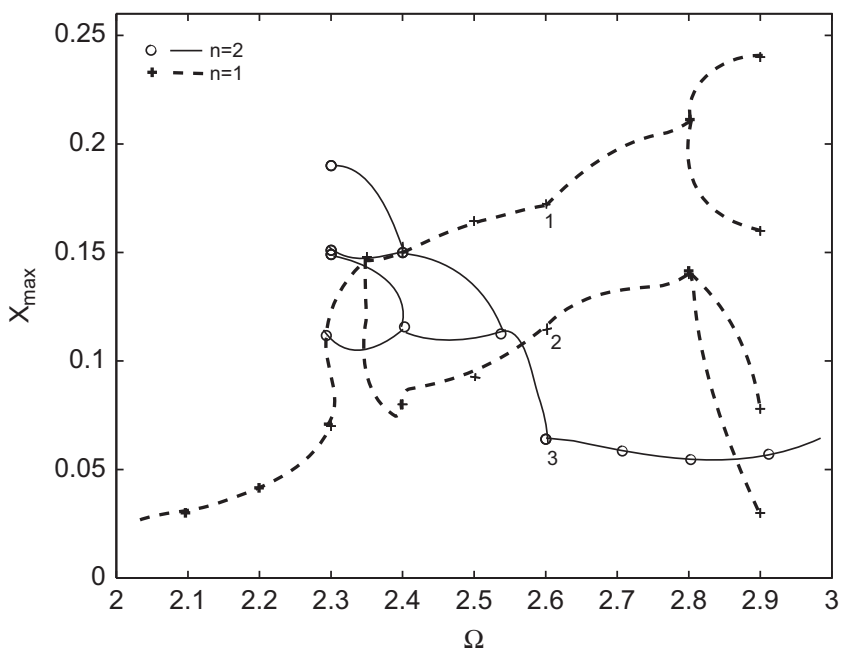

Fig. 5. The bifurcation diagram as $\Omega$ changes. $X_{\max }$ is the amplitude. The numbers of 1 and 2 are two period-1 motions whose time series and phase portraits are plotted in Fig. 6 . The number of 3 indicates a period- 2 motion whose time series and phase portraits are plotted in Fig. 7 a.

(a)

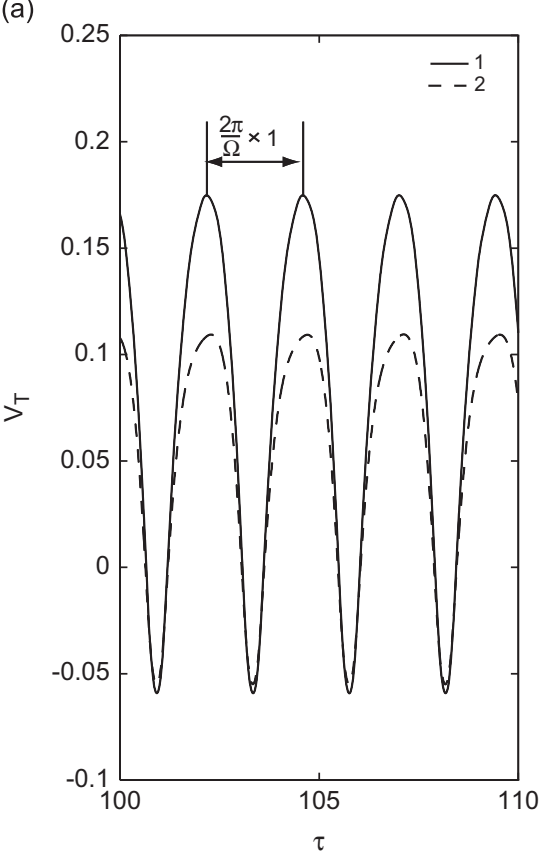

(b)

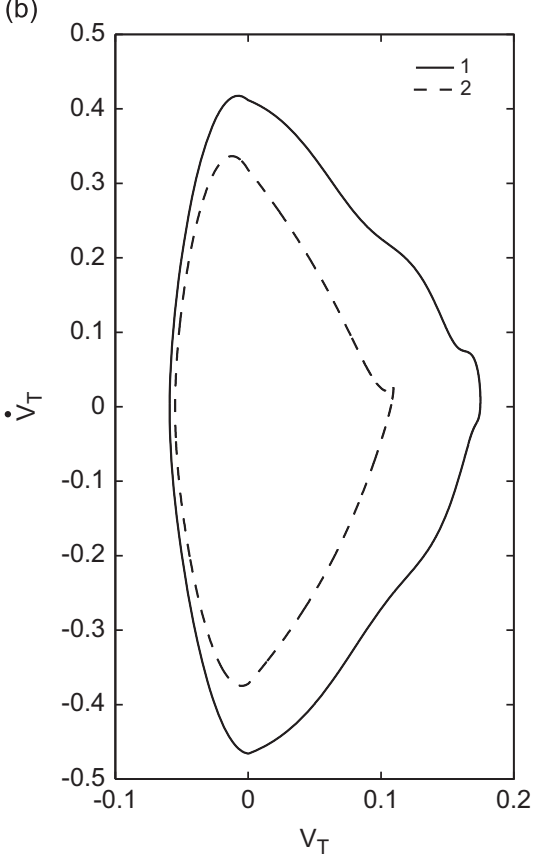

Fig. 6. The two period- 1 oscillations at $\Omega=2.6$ and $\alpha_{1}=0.1$. Solid and dashed lines correspond to the states marked as 1 and 2 in Fig. 5 . (a) Time series; (b) phase plane. 
(a)

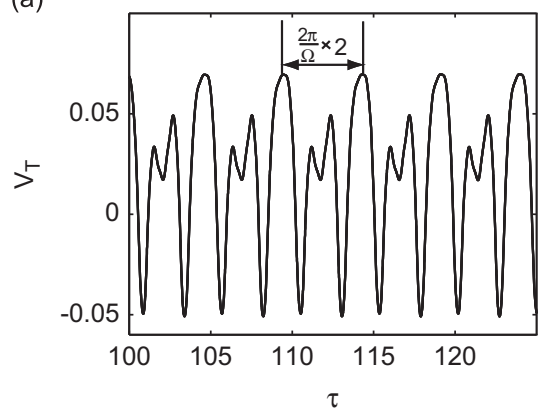

(c)

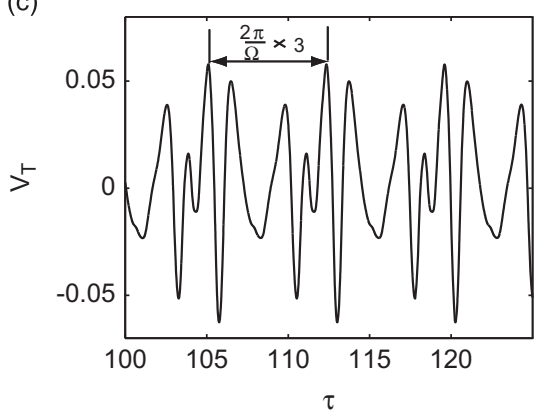

(b)

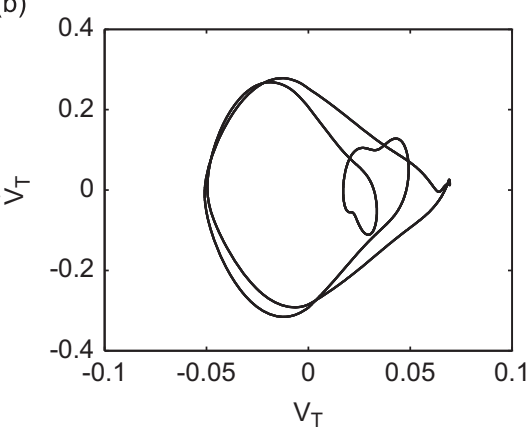

(d)

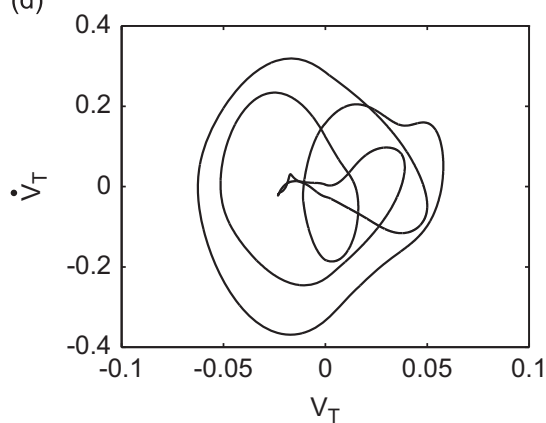

Fig. 7. The period-2 and period-3 motions at $\Omega=2.6$ and $\alpha_{1}=0.1$. (a) Time series and (b) phase plane of the period-2 motion. (c) Time series and (d) phase plane of the period-3 motion.
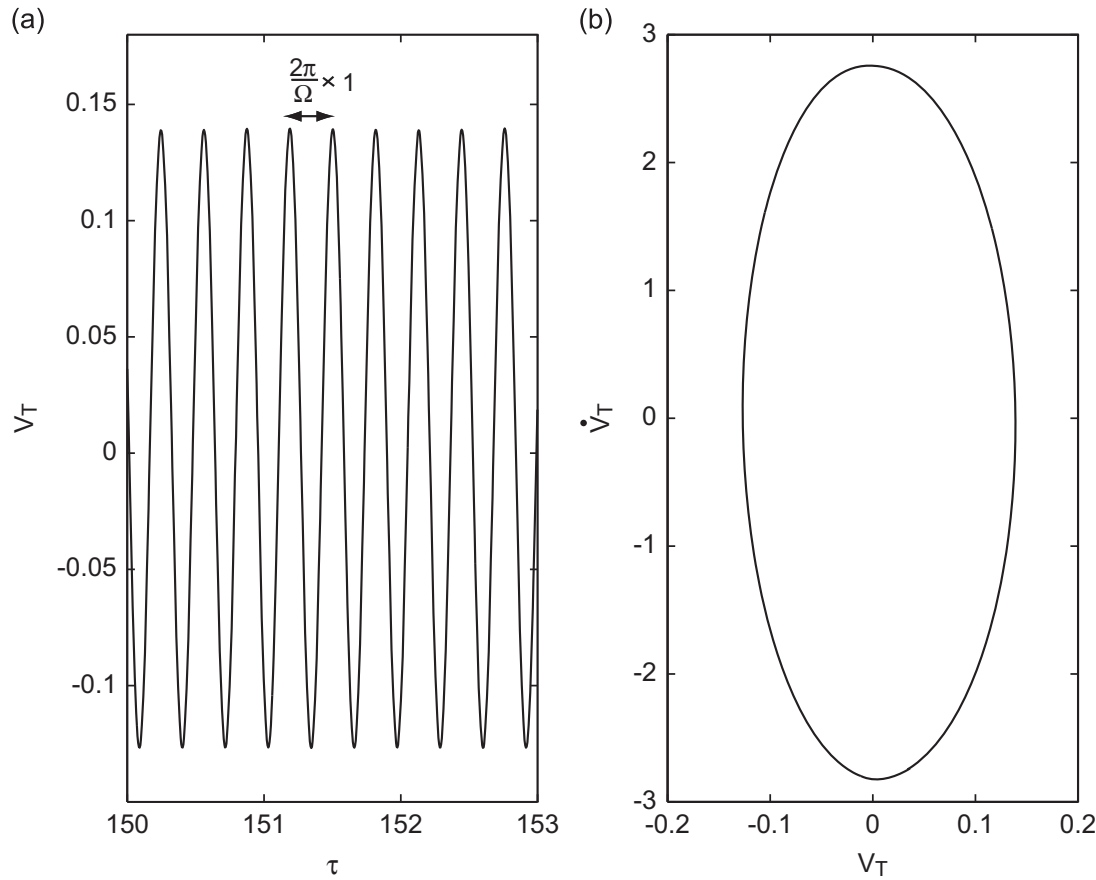

Fig. 8. The period- 1 motion at $\Omega=20$ and $\alpha_{1}=0.1$. (a) Time series; (b) phase plane.

The vibration of AFM in air has high $Q$ factor, or say small damping [14]. Therefore, here the relative small values are taken for $\alpha_{3}$ and $\alpha_{6}$ to make the system an underdamped one. Recently, both experiment and theory show that due to the fluctuating electromagnetic field between the AFM tip and sample, there is a noncontact friction force which is several orders of magnitude larger than the vdW friction and this noncontact force shares the same form as that of damping force [43]. Therefore, this noncontact friction force may also be incorporated in this model by choosing large $\alpha_{3}$. $\alpha_{4}$ defined 
(a)

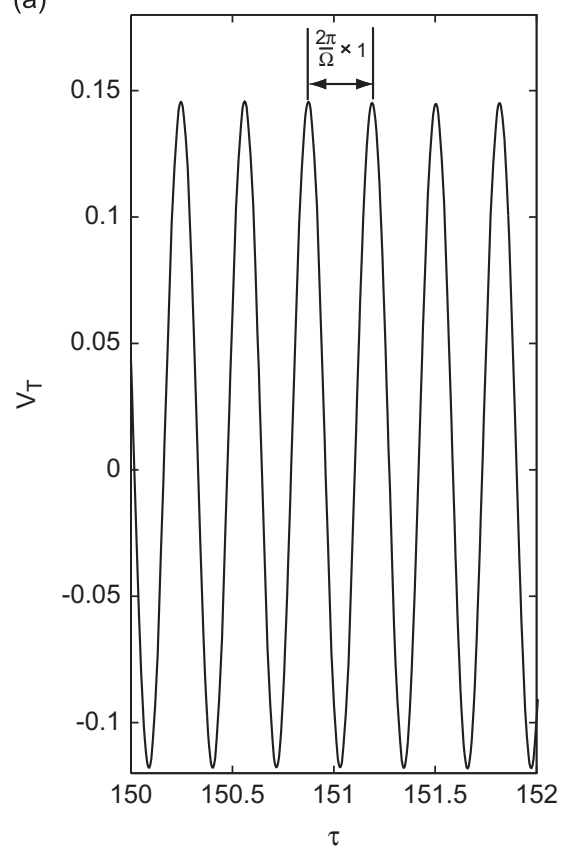

(b)

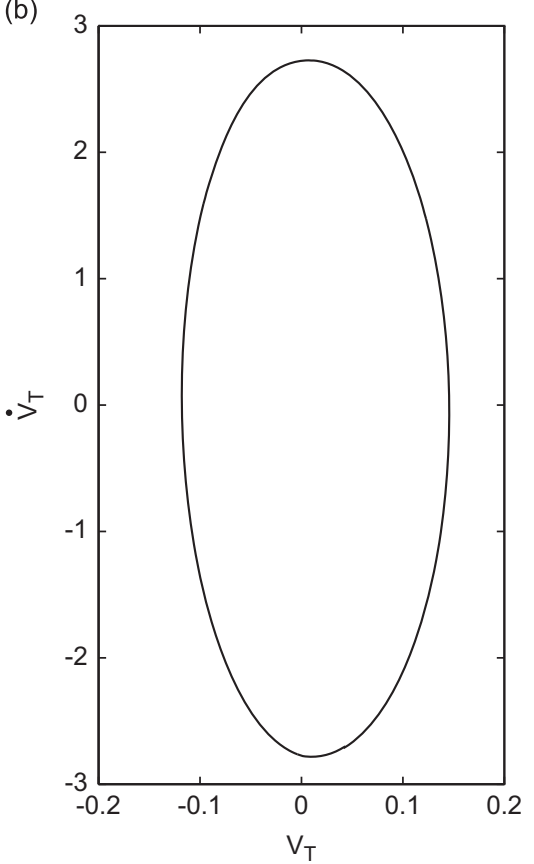

Fig. 9. Another period- 1 motion at $\Omega=20$ and $\alpha_{1}=0.1$. (a) Time series; (b) phase plane.

(a)

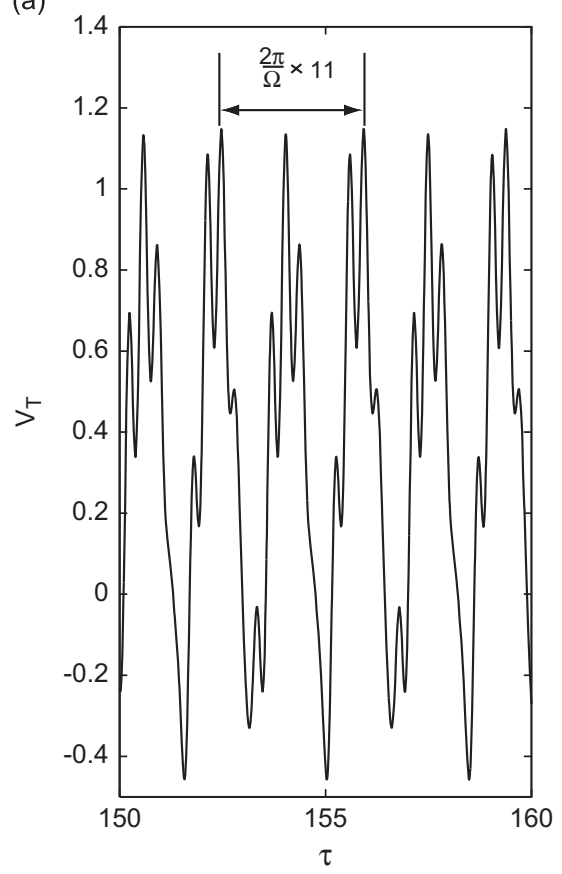

(b)

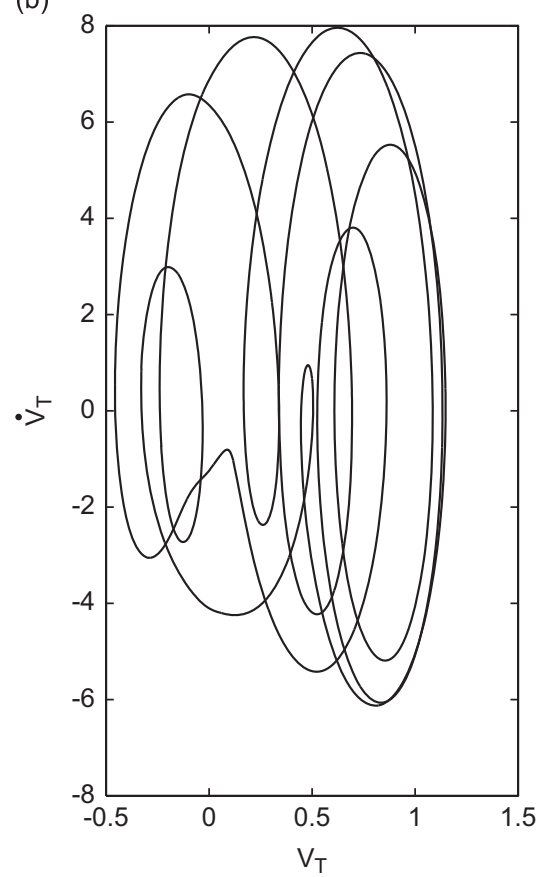

Fig. 10. The period- 11 oscillation at $\Omega=20$ and $\alpha_{1}=0.1$. (a) Time series; (b) phase plane.

in Eq. (7) indicates the ratio of the stiffness of the tip-sample contact to that of the AFM cantilever. The stiffness of the AFM cantilever is given as $K_{c}=3 E I / L^{3}[33,35]$ and the tip-sample contact stiffness can be calculated by Hertz model as $K_{1}=2 E^{*} \sqrt{R \Delta}\left(R\right.$ : the radius of tip, $\Delta$ is the indentation depth; $1 / E^{*}=\left(1-v_{1}^{2}\right) / E_{1}+\left(1-v_{2}^{2}\right) / E_{2}$; here $E_{1}, E_{2}$ and $v_{1}, v_{1}$ are Young's moduli and Poisson's ratios of the tip and sample, respectively) [33]. In [44], 10 different samples with $E_{2}$ ranging from $1 \mathrm{MPa}$ to $50 \mathrm{GPa}$ were indented by an AFM tip made of single crystal silicon $\left(E_{1}=130 \mathrm{GPa}\right.$ and $\left.v_{1}=0.27\right)$. Suppose $\Delta=R / 9, \alpha_{4}$ 's of these 10 materials can be calculated as $\alpha_{4}=K_{1} L^{3} /(E I)=3 K_{1} / K_{c}$, which range from $4.7 \times 10^{-3}$ to 233.5 . For 

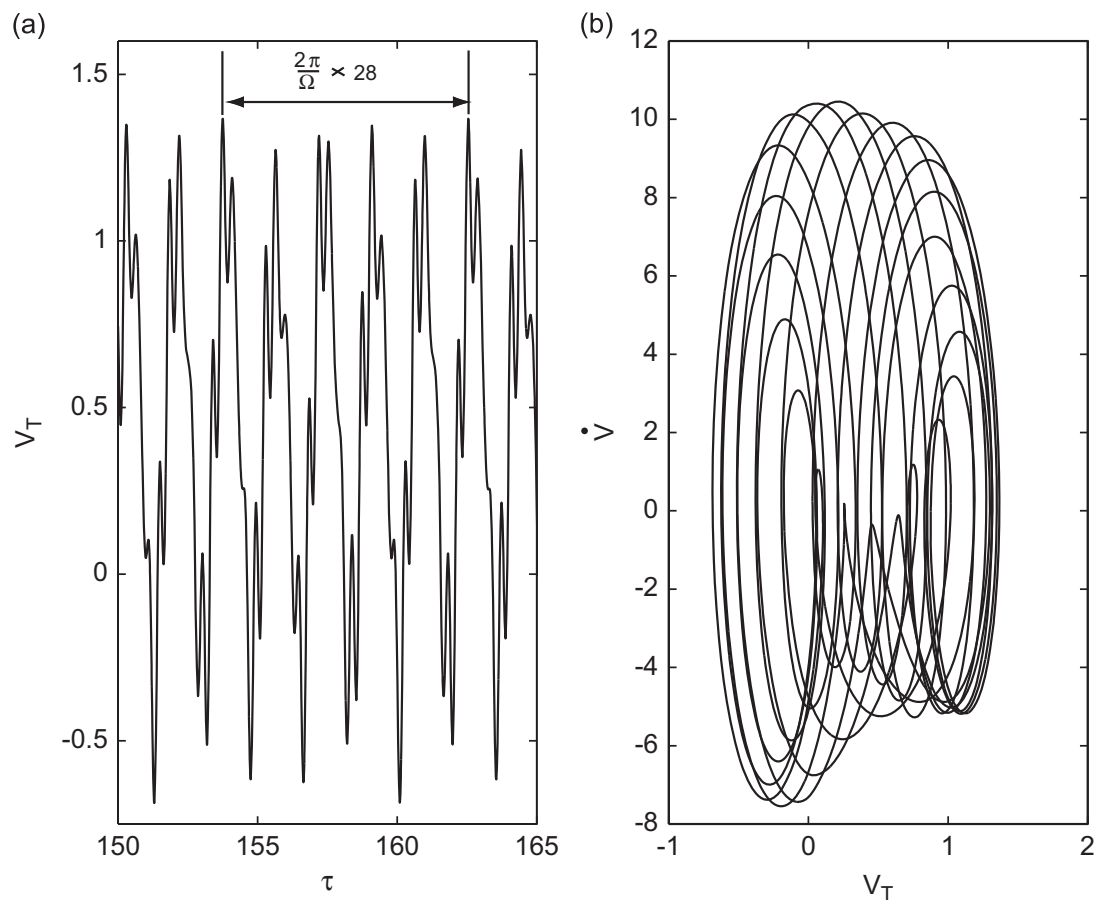

Fig. 11. The period-28 oscillation at $\Omega=20$ and $\alpha_{1}=0.1$. (a) Time series; (b) phase plane.

(a)

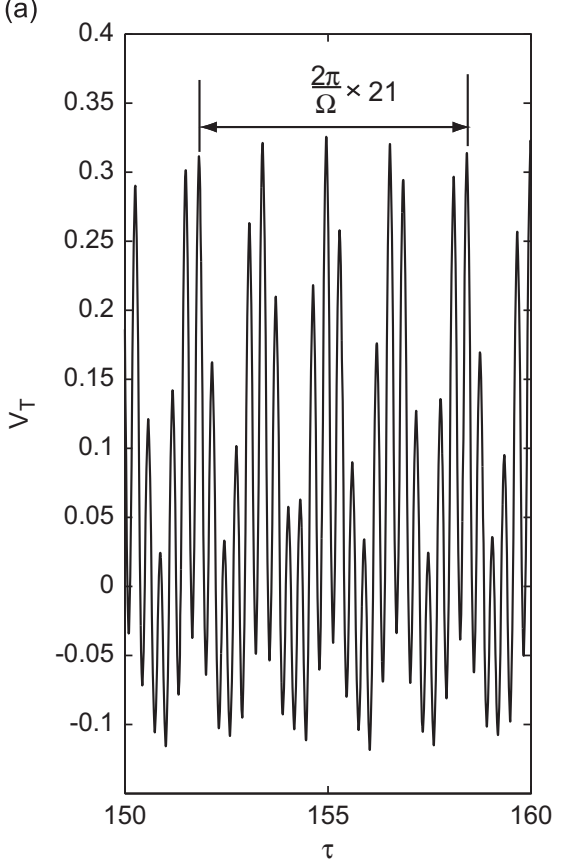

(b)

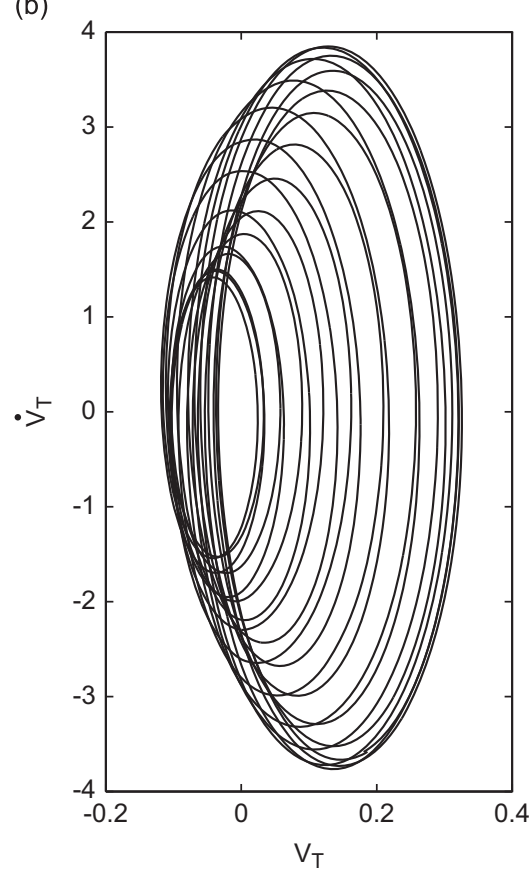

Fig. 12. The period-21 oscillation at $\Omega=20$ and $\alpha_{1}=0.1$. (a) Time series; (b) phase plane.

polystyrene ( $E_{2}=4 \mathrm{GPa}$ ) and highly oriented pyrolytic graphite ( $E_{2}=5 \mathrm{Gpa}$ ) [44], their $\alpha_{4}$ 's are 18.7 and 23.4 , respectively. As mentioned before, the tip mass can have significant impact on the intermittent contact dynamics by changing the system eigenfrequencies and coupling modes. In Figs. 5-15, $\alpha_{1}$ is selected as 0.1 to show its influence. In Figs. 16 and 17 , $\alpha_{1}=0$ is selected for a comparison study.

Fig. 5 shows the bifurcation of system as control parameter $\Omega$ changes. $X_{\max }$ is the tip amplitude at the steady-state. For the grazing impact, the Poincaré mapping can be analytically derived [3,23]. Therefore, the bifurcation and the transition 
(a)

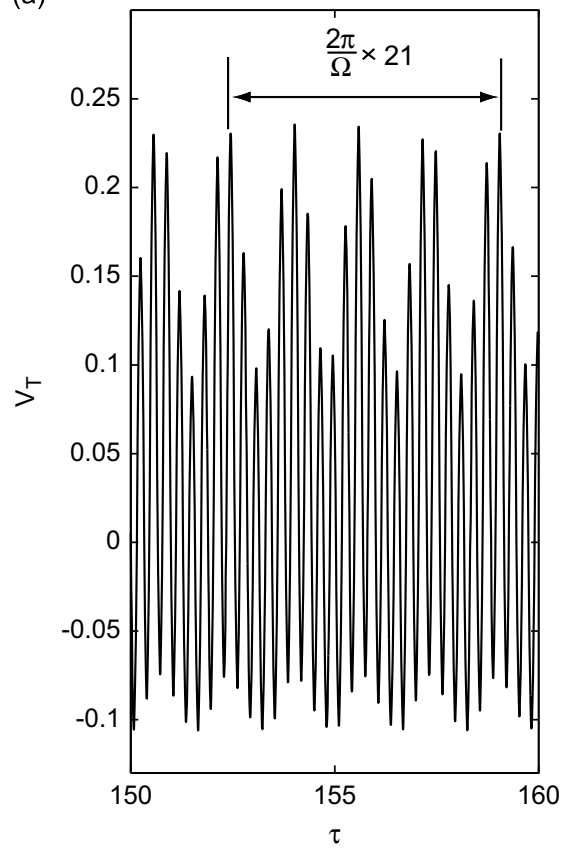

(b)

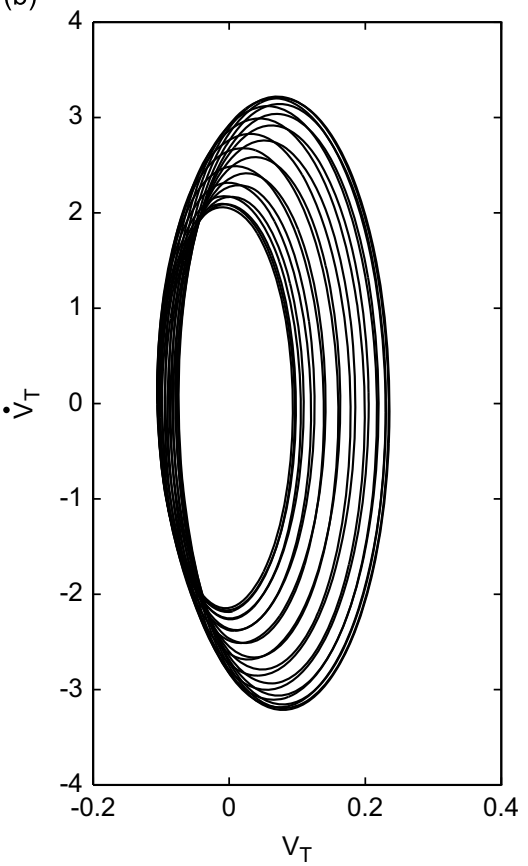

Fig. 13. Another period-21 oscillation at $\Omega=20$ and $\alpha_{1}=0.1$. (a) Time series; (b) phase plane.

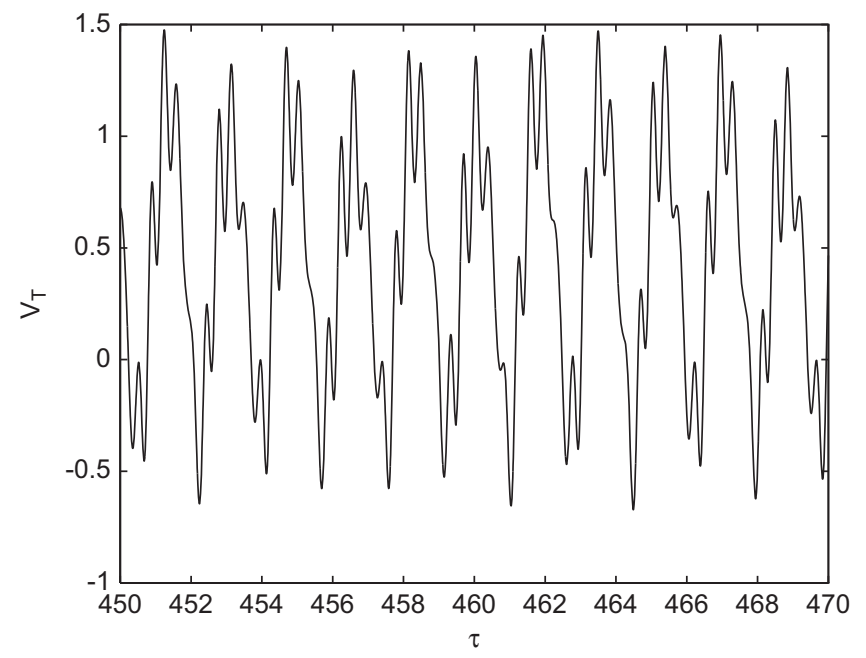

Fig. 14. The time series of a nonperiodic oscillation when $\Omega=20$ and $\alpha_{1}=0.1$.

to chaos can be clearly shown [3]. However, for the system with the tip mass modeled by the Dirac delta function and multi-modal analysis, to analytically derive the Poincaré mapping will be extremely difficult if not impossible [26]. In Figs. 5, period-1 and period-2 bifurcations are shown. While, other subharmonic motion like period-3 exists, which is not shown in Fig. 5. If the driving frequency further increases, nonperiodic motions will evolve from period- 1 motions. In the region of $2.0 \leq \Omega \leq 2.15$, nonperiodic motions evolving from period- 2 are also observed. But it is safe not to conclude that those nonperiodic motions are chaos. It may be transitional behavior as several period- $n$ motions coexist at one driving frequency. So the nonperiodic motion can be the transitional results that the motion of period- $m$ evolves to the motion of period- $n$, or the motion itself oscillates between the orbits even the time lasts long enough. It is also worth mentioning that in Shaw and Holmes's bifurcation diagram, they define a transitional zone and chaos is not shown, either [26]. It is noticed that the bifurcation diagram in Fig. 5 is very different from that of an impact oscillator [26].

Fig. 6 examines the time series and phase portraits of two period- 1 motions marked as 1 and 2 in the bifurcation diagram of Fig. 5. Both the motions of intermittent contact shown in both time series and phase portraits are asymmetric. The discontinuity of the spring stiffness and damping are responsible for the asymmetry and distortion. The time series and 
(a)

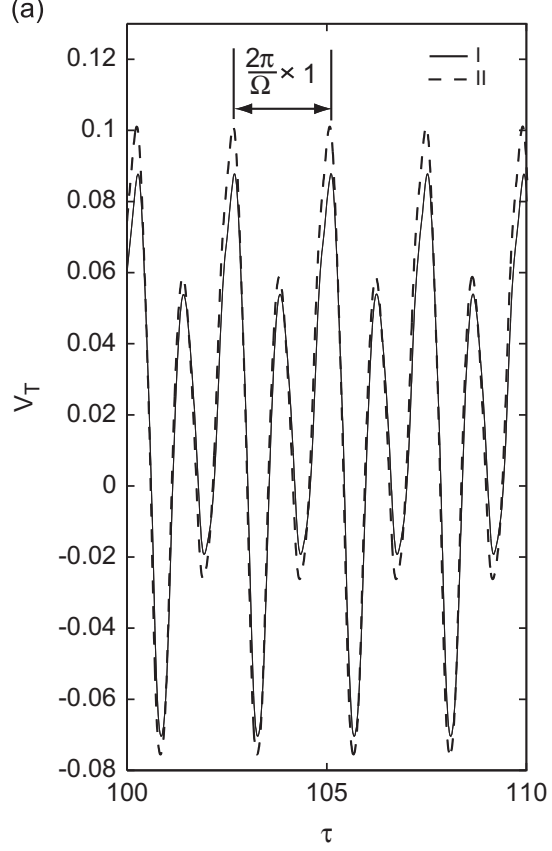

(b)

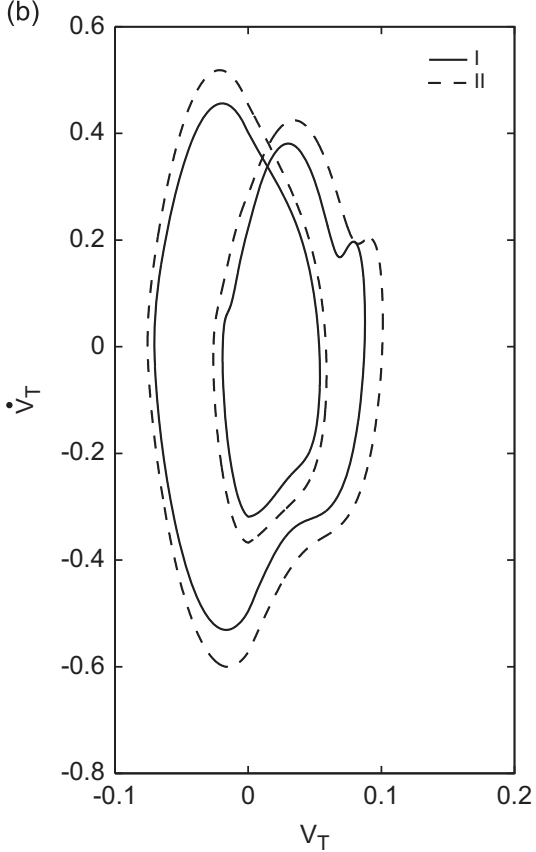

Fig. 15. Two period- 1 oscillations at $\Omega=20$ and $\alpha_{1}=0$. (a) Time series; (b) phase plane.

(a)

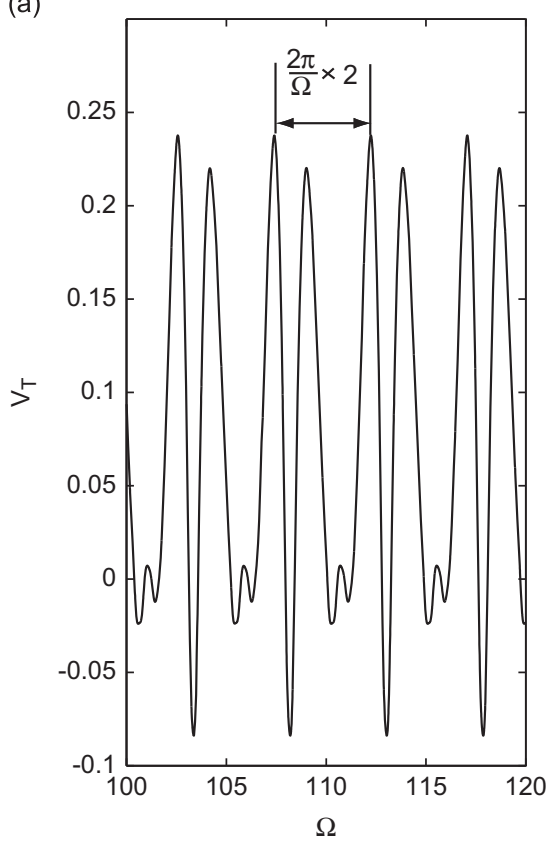

(b)

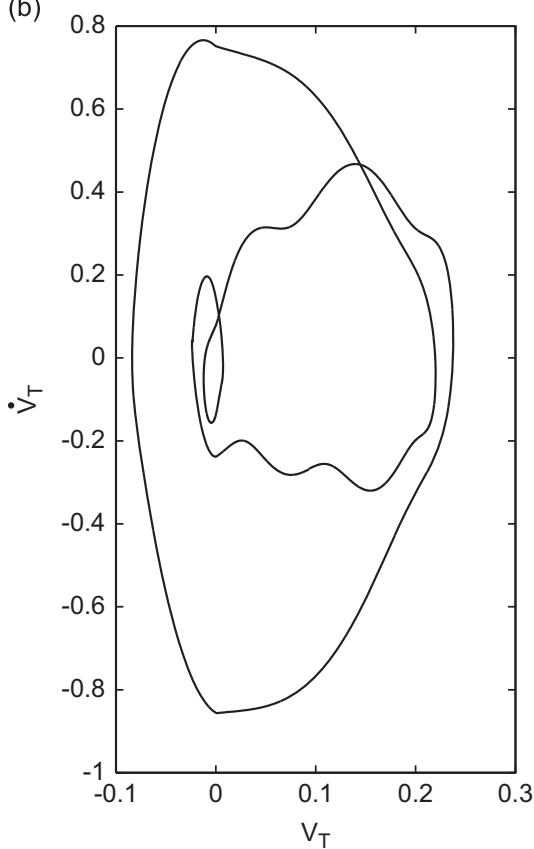

Fig. 16. The period- 2 oscillation at $\Omega=20$ and $\alpha_{1}=0$. (a) Time series; (b) phase plane.

phase portraits of the motion marked as 3 in Fig. 5 are shown in Fig. 7a and b. Besides period-1 and period-2 motions at $\Omega=2.6$, period-3 motion also exists. The time series and phase portrait of period-3 motion are presented in Fig. 7c and d.

Figs. 8 and 9 show the time series and phase portraits of two period- 1 motions at $\Omega=20$. As seen in Eq. (23), $\Omega=20$ is close to the second natural frequency. These two motions only have very small difference, which indicates the start of bifurcation and is also the reason why we put them in two separate plots. The amplitude is 0.145 in Fig. 8 and 0.148 in Fig. 9. Compared with the two period-1 motions at $\Omega=2.6$ in Fig. 6, these two period- 1 motions at $\Omega=20$ are much less asymmetric and phase portraits are less distorted. Besides these two period- 1 motions, other coexisting long period motions at $\Omega=20$ are also observed. Figs. 10 and 11 show the time series and phase portraits of period- 11 and period- 28 motions. Compared with those period- 1 motions, the 
(a)

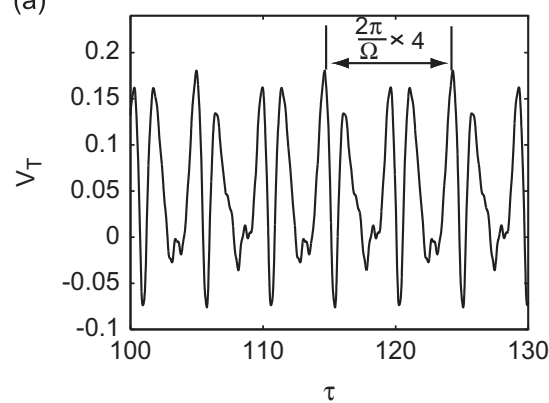

(c)

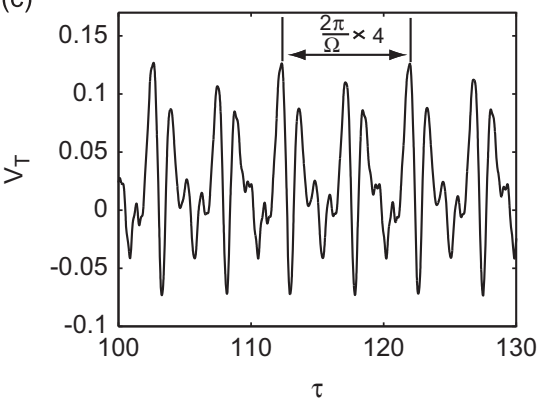

(b)

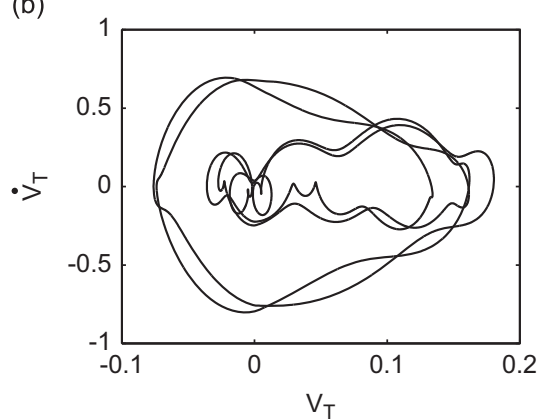

(d)

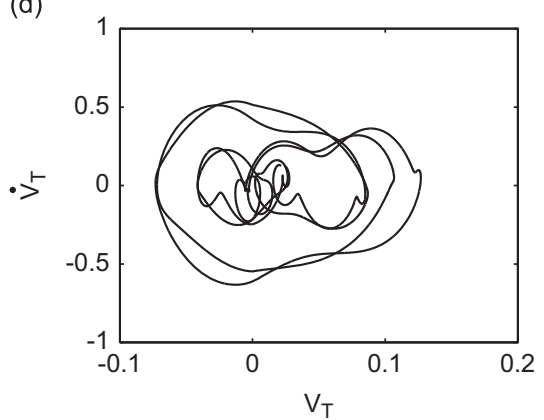

Fig. 17. Two period-4 oscillations at $\Omega=20$ and $\alpha_{1}=0$. (a) and (c) Time series; (b) and (d) phase planes.

maximum amplitudes of period-11 and period-28 are 1.18 and 1.45 , respectively. The asymmetry of their time series is severe and their phase portraits are greatly distorted. Two period-21 motions with $\Omega=20$ are also observed as shown in Figs. 12 and 13 . Their maximum amplitudes are 0.34 and 0.245 , respectively, which have the same order of magnitude of those period- 1 motions and much less than those of period-11 and period-28. The asymmetry of the two period-21 motions are also severe, while, their phase portraits are much less distorted. It is worth pointing out that the phase portraits of these two period-21 motions are very similar to those of an impact oscillator [26]. The nonperiodic motion as shown in Fig. 14 also exists at $\Omega=20$. Although it shows some pattern and looks like some long period motion, it just does not repeat its motion within the tolerance of computational error. It is noticed that the amplitude of this nonperiodic motion is close to those of period-11 and period-28.

It is indicated in Eq. (10) and Fig. 3 that the concentrated mass $\left(\alpha_{1}\right)$ physically causes the eigenfrequency change and mathematically couples the modes in the governing equation. For nonzero $\alpha_{1}$ case, it is very difficult to tell the separate contribution of eigenfrequency change and mode coupling to the dynamic behavior of the intermittent contact system. In Figs. 15-17, $\alpha_{1}$ is set to be zero. So mathematically, the modes derived from Eq. (10) are decoupled in the noncontact region. Fig. 15 shows two period- 1 motions at $\Omega=2.6$. The difference of these two period- 1 motions with those in Fig. 6 is very obvious. So is the period- 2 motion in Fig. 16 with that in Fig. 7a and b. We do not find the period-3 motion for $\alpha_{1}=0$ case at $\Omega=2.6$. However, period- 4 motion which is not found in $\alpha_{1}=0.1$ case exists in $\alpha_{1}=0$ case. Fig. 17 shows the time series and phase portraits of the two period-4 motions. For the $\alpha_{1}=0$ case, the mode coupling effect is gone. As $\alpha_{1}$ changes to zero, not only the eigenfrequencies change (thus the system response to the driving amplitude/frequency changes), but also the ratio of the eigenfrequencies before and after contact changes though the discontinuity of stiffness keeps the same. These two changes together result in the different dynamic behavior during the intermittent contact.

\section{Conclusion}

In the AFM intermittent contact dynamics, the primary effects are the frequency-amplitude response and intermittent contact. In this study, the sample is relatively soft and the system with intermittent shows much richer dynamic behavior than that of an impact oscillator. Various subharmonic and nonperiodic motions are observed. Some subharmonic motions are very similar to those of an impact oscillator; some subharmonics exhibit very different asymmetric motions and greatly distorted phase portraits. The bifurcation diagram of the system also shows the difference with that of an impact oscillator. The rich subharmonic patterns are obtained by changing driving frequency and initial conditions. These subharmonic motions can have significant impact on the interpretation of the experimental data obtained in the AFM tapping mode. The participation of the higher modes into the motion even for the relatively low driving frequency is also shown. The multi-modal analysis and convergence study are thus necessary. The tip mass effects on the whole system dynamic behavior are also shown. This analysis offers a more accurate and general approach to study the AFM intermittent contact dynamics, especially for the intermittent contact with a soft sample. 


\section{Acknowledgment}

This project is supported by the National Natural Science Foundation of China (NSFC nos. 10721202 and 11023001 ) and the Chinese Academy of Sciences (Grant no. KJCX2-EW-L03).

\section{References}

[1] G. Binnig, C. Quate, C. Gerber, Atomic force microscope, Physical Review Letters 56 (1986) 930-934.

[2] Q. Zhong, D. Inniss, K. Kjoller, V.B. Ellings, Fractured polymer/silica fiber surface studied by tapping mode atomic force microscopy, Surface Science Letters 290 (1993) L688-L692.

[3] W. Van de water, J. Molenaar, Dynamics of vibrating atomic force microscopy, Nanotechnology 11 (2000) 192-199.

[4] L. Nony, R. Boisgard, J.P. Aimé, Nonlinear dynamic properties of an oscillating tip-cantilever system in tapping mode, Journal of Chemical Physics 111 (1999) 1615-1627.

[5] A.E. Pelling, S. Sehati, E.B. Gralla, J.S. Valentine, J.K. Gimzewski, Local nanomechanical motion of the cell wall of Saccharomyces cerevisiae, Science 305 (2004) 1147-1150.

[6] N.A. Burham, A.J. Kulik, G. Gremaud, G.A.D. Briggs, Nanosubharmonics: the dynamics of small nonlinear contacts, Physical Review Letters 74 (1995) 5092-5095.

[7] A.B. Pippard, The Physics of Vibration, Cambridge University Press, Cambridge, 1978.

[8] N.A. Burham, O.P. Behrend, F. Oulevey, G. Gremaud, P.J. Gallo, D. Gourdon, E. Dupas, A.J. Kulik, H.M. Pollock, G.A.D. Briggs, How does a tip tap, Nanotechnology 8 (1997) 67-75.

[9] J. Berg, G.A.D. Briggs, Nonlinear dynamics of intermittent-contact mode atomic force microscopy, Physical Review B 55 (1997) R13381-R13384

[10] R. Garcia, A.S. Paulo, Dynamics of a vibrating tip near or in intermittent contact with a surface, Physical Review B 60 (2000) 14899-14908.

[11] J.P. Aimé, R. Boisgard, L. Nony, G. Couturier, Nonlinear dynamic behavior of an oscillating tip-microlever system and contrast at the atomic scale, Physical Review Letters 82 (1999) 3388-3391.

[12] S.I. Lee, S.W. Howell, A. Raman, R. Reifenberger, Nonlinear dynamics of microcantilevers in tapping mode atomic force microscopy: a comparison between theory and experiment, Physical Review B 66 (2002) 115409.

[13] P. Attard, J.C. Schulz, M.W. Rutland, Dynamic surface force measurement. I. van der Waals collision, Review of Scientific Instruments 69 (1998) 3852-3866.

[14] S. Rützel, S.I. Lee, A. Raman, Nonlinear dynamics of atomic-force-microscope probes driven in Lennard-Jones potentials, Proceedings of the Royal Society (London) A 459 (2003) 1925-1948.

[15] D. Martinez-martin, E.T. Herruzo, C. Dietz, J. Gomez-herrero, R. Garcia, Noninvasive protein structural flexibility mapping by bimodal dynamic force microscopy, Physical Review Letters 106 (2011) 198101.

[16] M. Stark, R.W. Stark, W.M. Heckl, R. Guckenberg, Spectroscopy of the anharmonic cantilever oscillations in tapping mode atomic-force microscopy, Applied Physics Letters 77 (2000) 3293-3295.

[17] B. Gotsmann, C. Seidel, B. Anzykowski, H. Fuchs, Conservative and dissipative tip-sample interaction forces probed with dynamic AFM, Physical Review B 60 (1999) 11051-11061.

[18] J.A. Wickert, M.D. Mote, Transverse vibration of axially moving string-particle system, Journal of the Acoustic Society of America 84 (1988) 963-969.

[19] K.D. Murphy, Y. Zhang, Vibration and stability of a cracked translating beam, Journal of Sound and Vibration 237 (2000) 319-335.

[20] P.V. Bayly, K.D. Murphy, Coupling between dissimilar modes in an asymmetrically forced string, Journal of the Acoustic Society of America 103 (1998) 3362-3369.

[21] S.W. Shaw, P.J. Holmes, Periodically forced linear oscillator with impacts: chaos and long-period motions, Physical Review Letters 51 (1983) 623-626.

[22] Y. Zhang, K.D. Murphy, Static and dynamic structural modeling analysis of atomic force microscope, in: B. Bhushan (Ed.), Scanning Probe Microscopy in Nanoscience and Nanotechnology, Springer, Berlin, 2010 (Chapter 8).

[23] A.B. Nordmark, Non-periodic motion caused by grazing incidence in an impact oscillator, Journal of Sound and Vibration 145 (1991) $279-297$.

[24] J.M.T. Thompson, H.B. Stewart, Nonlinear Dynamics and Chaos, John Wiley \& Sons, Inc., New York, 1991.

[25] P.J. Holmes, The dynamics of repeated impacts with a sinusoidally vibrating table, Journal of Sound and Vibration 84 (1982) $173-189$.

[26] S.W. Shaw, P.J. Holmes, A periodically forced piecewise linear oscillator, Journal of Sound and Vibration 90 (1983) 129-155.

[27] S.W. Shaw, Forced vibration of a beam with one-sided amplitude constraint: theory and experiment, Journal of Sound and Vibration 99 (1985) 199-212.

[28] K.D. Murphy, T.M. Morrison, Grazing instabilities and post-bifurcation behavior in an impacting string, Journal of the Acoustic Society of America 111 (2002) 884-892.

[29] M.D. Todd, L.N. Virgin, An experimental impact oscillator, Chaos, Solitons and Fractals 8 (1997) 699-714.

[30] H. Hölscher, W. Allers, U.D. Schwarz, A. Schwarz, R. Wiesendanger, Determination of tip-sample interaction potentials by dynamic force spectroscopy, Physical Review Letters 83 (1999) 4780-4783.

[31] F.J. Giessibl, Forces and frequency shifts in atomic-resolution dynamic-force microscopy, Physical Review B 56 (1997) 16010-16015.

[32] W.H. Lin, Y.P. Zhao, Nonlinear behavior for nanoscale electrostatic actuators with Casimir force, Chaos, Solitons and Fractals 23 (2005) $1777-1785$.

[33] Y. Zhang, Extracting nanobelt mechanical properties from nanoindentation, Journal of Applied Physics 107 (2010) 123518

[34] S. Santos, N.H. Thomson, Energy dissipation in a dynamic nanoscale contact, Applied Physics Letters 98 (2011) 013101.

[35] Y. Zhang, K.D. Murphy, Response of a finite beam in contact with a tensionless foundation under symmetric and asymmetric loading, International Journal of Solids and Structures 41 (2004) 6745-6758.

[36] T.C. Chang, R.R. Craig, Normal modes of uniform beams, Journal of Engineering Mechanics 95 (1969) 1027-1031.

[37] W.H. Press, B.P. Flannery, S.A. Teukolsky, W.T. Vettering, Numerical Recipes, Cambridge University Press, Cambridge, 1986.

[38] P.V. Bayly, L.N. Virgin, Chaotic rattling of a piecewise nonlinear oscillator, 91-WA-DSC-17, ASME Winter Annual Meeting, Atlanta, GA, December 1-6, 1991.

[39] L. Meirovitch, Computational Method and Structural Dynamics, Sijthoff \& Noordhoff, Maryland, 1980.

[40] L.N. Virgin, Introduction to Experimental Nonlinear Dynamics, Cambridge University Press, Cambridge, 2000.

[41] A.H. Nayfeh, D.T. Mook, Nonlinear Oscillations, John Wiley \& Sons, Inc., New York, 1979.

[42] M.S. El Naschie, Stress, Stability and Chaos in Structural Engineering: An Energy Approach, McGraw-Hill Book Company, London, 1990.

[43] A.I. Volokitin, N.J. Persson, Adsorbate-induced enhancement of electrostatic noncontact friction, Physical Review Letters 94 (2005) 086104.

[44] O. Sahin, N. Erina, High-resolution and large dynamic range nanomechanical mapping in tapping-mode atomic microscopy, Nanotechnology 19 (2008) 445717. 